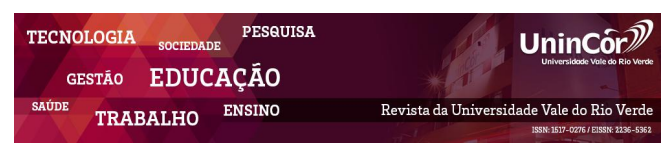

Revista da Universidade Vale do Rio Verde ISSN: 1517-0276 / EISSN: 2236-5362 v. 17 | n. 2 | Ano 2019

Tereza Cristina dos Reis Ferreira Fisioterapeuta e professora da Universidade do Estado do Pará reis.tereza@gmail.com

João Pedro dos Reis da Costa

Graduando do curso de bacharelado em Medicina da Universidade do Estado do Pará jprcjprc29@gmail.com

Beatriz Sayuri Vieira Ishigaki Graduanda do curso de bacharelado em Medicina da Universidade do Estado do Pará beatrizishigaki00@gmail.com

Eduardo Augusto Silva Monteiro Graduando do curso de bacharelado em Medicina da Universidade do Estado do Pará eduardo.silvamonteiro93@gmail.com

\section{EFEITO DO USO DE PLANTAS MEDICINAIS EM MODELOS EXPERIMENTAIS DE CICATRIZAÇÃO}

\begin{abstract}
RESUMO
Os ferimentos cutâneos são problemas que ocorrem normalmente durante toda a vida de qualquer pessoa, principalmente pelo papel de órgão de barreira que a pele desempenha, isto acaba fazendo com que ela seja comumente lesionada, essas lesões, dependendo do paciente em que ocorrem, podem acabar tendo o seu processo de cicatrização retardado o que acarreta no desenvolvimento de uma úlcera crônica, neste sentido, o tratamento tradicional acaba sendo oneroso para o sistema de saúde, sendo então possível o emprego de plantas medicinais como alternativa para o tratamento dessas lesões. Nesse sentido, o objetivo da presente revisão de literatura é elencar o feito das plantas medicinais sobre o processo de cicatrização. Para isso foi realizada a busca de estudos nas bases de dados do PUBMED, LILACS e SCIELO com a utilização dos descritores Wound Healing, skin e Rats. Foram encontrados 66 artigos que se adequavam à temática da revisão, sendo o n final 20 artigos. A partir desses artigos, foi possível perceber que o processo de cicatrização é complexo e pode ser estimulado de variadas forma, aumentando a capacidade antioxidante da ferida, aumentando a quantidade de colágeno, hidroxiprolina, além de também evitar a ocorrência de infecções, todos esses fatores contribuem para que o tempo de contração da ferida diminuem, proporcionando desta maneira uma melhor cicatrização. Logo, há variadas plantas medicinas que já foram aplicadas em modelos experimentais de cicatrização, porém mais estudos poderiam ajudar com que estas plantas fossem empregadas na prática clínica.
\end{abstract}

Palavras-chave: Cicatrização. Plantas Medicinais. Modelos Animais.

\section{Effect of medicinal plants in experimental models of wound healing}

\begin{abstract}
Skin wounds are problems that are particularly difficult throughout life, mainly by the role of barrier organ that the skin plays, which makes the impression is commonly the process of delayed healing entails in the development of an eye ulcer, being now the dream of being medicated as an alternative for the treatment of the lesions. In this sense, the present literature review is to list the healing of medicinal plants on the healing process. Abstract This is a search of data in PUBMED, LILACS and SCIELO databases using a Wound Healing, skin and Rats descriptor. We found 66 articles that fit the theme of the review, in a way that only 20 articles were studied. From the articles, it was possible to perceive that the cicatrization process is complex and can be stimulated in several ways, increasing the antioxidant capacity of the wound, increasing the amount of collagen, hydroxyproline, besides being able to avoid infection, all these factors. Contribute so that the time of contraction of the wound decreases, thus providing a better wound healing. Therefore, there are more medicinal plants that have already been applied in
\end{abstract}


experimental healing experiments, since the studies were more effective with respect to the machines that were used in clinical practice.

Keywords: Wound Healing. Medicinal Plants. Animal Models.

\section{INTRODUÇÃO}

Ferimentos cutâneos consistem na formação de uma solução de continuidade na anatomia da epiderme, esse tipo de problema é muito comum durante toda a vida (SUGUMAR et al., 2014), podendo ocorrer por diversos mecanismos como a estase venosa, o trauma causado por um acidente ou por uma cirurgia.

A cicatrização desses ferimentos é um processo que compreende quatro fases: a fase hemostática, a inflamatória, a proliferativa e a de remodelação tecidual. Essas quatro fases devem ocorrer na ordem adequada e no tempo correto, sendo que interrupções nesse processo podem acabar gerando lesões que apresentam cicatrização retardada ou mesmo que não chegam a curar pelo fato de estarem estagnadas em uma determinada fase (GUO; DIPIETRO, 2010).

Durante esse processo de cicatrização diversos fatores podem afetá-lo de maneira a impossibilitar a formação de uma cicatriz adequada, esses fatores podem ser classificados em locais - como a oxigenação, presença de infecção e suficiência venosa - e fatores sistêmicos como a presença de uma doença de base, tal qual o Diabetes Mellitus, a idade do paciente, as medicações que este consume e o estado imunológico do mesmo. O manejo dessas injúrias é uma das principais preocupações quanto ao cuidado clínico das feridas cutâneas (GUO; DIPIETRO, 2010; EMING; MARTIN; TOMIC-CANIC, 2014).
Nesse contexto o principal exemplo de ferimento são as úlceras, que podem ser classificadas em agudas e crônicas, as últimas apresentam um aumento do número de casos nos últimos anos, principalmente em pessoas com idade superior a 65 anos, e geralmente a injúria começa com pequenas lesões causadas por picadas de insetos e coçaduras na pele seca (DEMDOVARICE; HAMBLING; HERMAN, 2012).

Outra forma de classificar esse tipo de úlcera pode ser em úlceras de pressão, úlceras diabéticas, úlceras venosas e úlceras de insuficiência arterial, cada uma com fatores desencadeantes distintos, além de apresentarem uma abordagem clínica distinta entre si (EMING; MARTIN; TOMIC-CANIC, 2014; JÄRBRINK et al., 2017).

Além disso, as úlceras crônicas ainda apresentam um comprometimento do estado geral do paciente, pelo fato desta continuar na fase inflamatória de cicatrização por um longo tempo, isso acaba refletindo em um estado de dor contínua, além de que em alguns casos essas úlceras podem nem mesmo vir a cicatrização, diminuindo ainda mais a qualidade de vida do paciente (IQBAL et al., 2017).

Mas não somente o paciente é afetado por esse tipo de injúria, o próprio sistema de saúde acaba sendo onerado, alguns estudos apontam que os gastos econômicos são substanciais com o cuidado dessas úlceras, de modo que medidas voltadas para a prevenção 
desse ocorrido seriam uma medida de impacto positivo sobre os sistemas de saúde, pois diminuiria a necessidade desse tipo de atendimento (CHAN et al., 2017; NUSSBAUM et al., 2018).

Nesse sentido, as plantas medicinais podem ser uma alternativa para o tratamento dessas úlceras, principalmente pelo fato de que elas já são empregadas em várias culturas tradicionais da África e da América com a finalidade de tratar feridas presentes na pele (TSALA et al., 2015).

Apesar de muito utilizadas pelo senso comum para $\mathrm{o}$ tratamento de várias enfermidades, o uso de plantas medicinais ainda conta com poucos estudos científicos que comprovem seus reais efeitos e diminuem a incerteza quanto a eficácia e a segurança do uso desse tipo de abordagem (TABANDEH; ORYAN; MOHAMMADALIPOUR, 2014).

O objetivo deste estudo é fazer uma revisão acerca do efeito das plantas medicinais sobre os modelos de cicatrização de modelos experimentais.

\section{MATERIAL E MÉTODOS}

Este estudo consiste em uma revisão integrativa da literatura, foi realizada a busca de artigos empregando-se as bases de dados PUBMED, LILACS e SCIELO, os descritores empregados foram Wound healing, skin e rats, entre os descritores foi usado o operador booleano AND. Como critério de inclusão foi estabelecido que os artigos que estivessem escrito nas línguas portuguesa e inglesa, que haviam sido publicados nos últimos 5 anos e que estivessem de acordo com os objetivos do presente estudo.
Ao analisar o total de artigos obtidos pelo mecanismo de pesquisa, apenas 66 estudos se encaixavam nos critérios de inclusão e foram incluídos nesta revisão apenas 20 artigos do total.

\section{REVISÃO DE LITERATURA}

\subsection{Modelos experimentais de cicatrização}

Há dois tipos de estudos realizados quanto a análise do processo de cicatrização, são eles: o modelo excisional e o modelo incisional, sendo distintas as análises realizadas em cada um destes (MOGHADAMTOUSI et al., 2015).

\subsubsection{Modelo Excisional}

O modelo excisional consiste na remoção de fragmento de pele por meio da secção do plano da epiderme com o da derme, dessa maneira, uma solução de continuidade é criada semelhante ao que acontece em uma úlcera (KOVÁČ et al., 2015; BEGASHAW et al., 2017).

Um desses modelos que é empregado para o estudo da cicatrização consiste em realizar uma demarcação da área a ser removida na própria pele do animal, para isso é utilizado um punch de diâmetro variável, posteriormente à demarcação, é utilizada uma lâmina de bisturi ou uma tesoura a fim de realizar a retirada do fragmento de tecido epitelial que foi delimitado no início do procedimento (ERGENE et al., 2017).

Outro método empregado para realizar a análise do processo cicatricial é a demarcação da porção de pele a ser retirada por meio de uma tesoura ou lâmina de bisturi e depois é feita a retirada do fragmento destacado pela separação do tecido dos planos mais profundos por meio da 
divulção do tecido subepitelial (KOVÁČ et al., 2015; CHANDRA et al., 2015; ERGENE et al., 2017; BHANDIRGE et al., 2015).

Esses modelos são empregados principalmente para fazer a análise da taxa de contração das bordas da ferida, que acaba sendo o reflexo de todo o processo antioxidante das plantas, além da atividade estimulante para a produção de colágeno e hidroxiprolina.

\subsubsection{Modelo Incisional}

O modelo incisional consiste em criar um ferimento inciso, ou seja, uma ferida linear no dorso do animal com a utilização de uma lâmina de bisturi e então fazer a aproximação das bordas por meio de sutura (TÜMEN et al., 2018).

O principal estudo realizado com esse modelo experimental é o de força de estiramento da cicatriz, de maneira a avaliar se a pele continua com a mesma resistência ao estiramento por meio de um tensiomêtro, de modo que é realizada uma comparação entre o grupo que recebe a intervenção e o grupo controle e as mudanças na força de estiramento é dada em relação de porcentagem (ÖZBILGIN et al., 2018; TÜMEN et al., 2018).

\subsection{Efeito das plantas na cicatrização}

Os efeitos das plantas medicinais sobre o processo de cicatrização são muito variáveis, sendo que os principais impactos estão relacionados com a atividade antioxidante, a contração da ferida, a produção de colágeno

\subsubsection{Atividade antioxidante}

Para a determinação da capacidade antioxidante os marcadores utilizados foram as enzimas Superóxido Dismutase (SOD), Catalase
(CAT) e Glutationa Peroxidase (GPX), essas enzimas apresentam uma grande relevância para o processor de cicatrização pois durante este processo ocorre a isquemia do tecido epitelial pela resposta inflamatória decorrente dos leucócitos, isso acarreta em produção de espécies reativas de oxigênio, que quando produzida em elevadas quantidades pode acarretar em estresse oxidativo e leva a formação de uma cicatriz disforme, dessa maneira, quando ocorre um aumento da capacidade antioxidante do tecido é possível que este se proteja dos danos celulares que os radicais livres podem causar. No caso do Bredo (Trianthema portulacastrum) houve um aumento da capacidade antioxidativa do grupo que recebeu a pomada de Bredo em relação ao grupo que não teve nenhuma intervenção, além disso, a concentração da pomada teve uma influência no resultado, de modo que quanto maior a sua concentração maior era o aumento das enzimas (MOGHADAMTOUSI et al., 2015; YADAV et al., 2017).

Além disso, outra planta também utilizada para tratar os ferimentos cutâneos e que apresentava influência sobre a oxidação do tecido em questão foi a graviola (Annona muticata), esta apresentou um aumento da atividade da enzima SOD, o que indica um efeito positivo no sentido de promover a cura das feridas, além disso, a CAT também teve aumento relevante nos grupos tratados com a graviola, indicando o mesmo efeito que a SOD apresentou, de modo a aumentar a velocidade de cicatrização (MOGHADAMTOUSI et al., 2015).

Outra análise também realizada para averiguar a capacidade antioxidante da planta é a estimativa da Malondialdeído (MDA), que consiste em um marcado de estresse oxidativo, o 
que indica a formação de espécies reativas de oxigênio no tecido em análise, quando esta enzima se apresenta elevada, há um sinal de que as defesas antioxidantes do organismo falharam. No estudo realizado por Panda (2014), foi analisada a atividade da Typha elephantina foi possível perceber que esta teve uma diminuição dos níveis de MDA em relação ao grupo controle, indicando para uma atividade antiperoxidação lipídica. A atividade que a $T$. elephantina teve pode ser explicada por causa da presença de quercetina e flavonoides em sua composição.

\subsubsection{Contração da ferida}

Plantas como a bombardeira (Calotropis procera) e a amendoeira-da-praia (Terminalia catappa) apresentaram resultados positivos quanto ao tempo total de cicatrização e quanto à porcentagem de cura da ferida, ambas plantas demonstraram resultado superior ao grupo controle que em um dos experimentos recebeu antisséptico e no outro recebeu corticoide (KHAN et al., 2014; TSALA et al., 2015).

Outro estudo em que o Feno-Grego foi utilizado para auxiliar na cicatrização, o grupo de animais que o recebeu apresentou já no $14^{\circ}$ dia de experimento o completo fechamento da ferida que havia sido realizada, enquanto que os demais grupos não chegaram a completar o processo de cicatrização dentro do período estabelecido, a análise desse estudo também foi feita empregando-se a coloração da ferida, foi possível perceber que o grupo que não recebeu nenhuma intervenção apresentava uma ferida de coloração avermelhada, enquanto que o grupo que recebeu o Feno-Grego logo evoluiu para a coloração marrom, indicando para a formação de coágulo sanguíneo e depois evoluiu para a formação de tecido de granulação com cor rosa (KTARI et al., 2016).

Já a Cleome viscosa apresentou um resultado semelhante à Gentemicina, uma vez que ambas apresentaram uma taxa de retração da ferida de aproximadamente $90 \%$ no $21^{\circ}$ dia de experimento, por outro lado, quando comparado com o grupo que não recebeu nenhuma intervenção, houve uma diferença estatística significante entre os grupos, uma vez que, no grupo controle no $21^{\circ}$ dia a ferida havia cicatrizado em torno de 50\% (UPADHYAY et al., 2014).

\subsubsection{Força de ruptura}

Outro achado interessante está relacionado com a força de ruptura da cicatriz induzida no modelo experimental de incisão, a planta Typha elephantina, presente principalmente na África e na Ásia, apresentou resultados melhores do que o grupo controle com relação a força de ruptura da cicatriz, sendo que este efeito estava ligado diretamente com a concentração do extrato utilizado. Esse fato pode ser explicado por meio do estímulo que essa planta faz para a produção de colágeno e estabilizando as fibras entre si, desse modo, a ferida tem o processo de cicatrização estabilizado e a cura acaba ocorrendo mais rapidamente (PANDA; THAKUR, 2014).

\subsubsection{Conteúdo de hidroxiprolina}

A Hidroxiprolina foi estimada nos tecidos de granulação dos modelos de ferida de excisão, como no estudo utilizando Caesalpinia mimosoides, no qual foi aplicado o método Bergman e Loxley (1961). Nesta pesquisa, a 
proporção de hidroxiprolina encontrada tanto nos estratos aquosos quanto etanólicos foram significativamente maiores (91.55 $\pm 0.27 \mu \mathrm{g} / 100 \mathrm{mg}$ e $68.44 \pm 0.59 \mu \mathrm{g} / 100 \mathrm{mg}$, $\mathrm{p} \leq 0.000$ ) que as encontradas no grupo controle, evidenciando o poder cicatrizante do extrato, visto que a hidroxiprolina é responsável pelo aumento da proliferação e migração de fibroblastos, com consequente deposição de colágeno, com papel vital na rápida contração da ferida e menor tempo de reepitelização desta (BHAT et al., 2016).

Outro estudo que determinou o nível de hidroxiprolina em tecido cicatrizante utilizou-se da Alocasia denudata. Nesta análise verificou-se que o teor de hidroxiprolina atingiu um nível mínimo no $10^{\circ}$ dia, enquanto que o pico foi no $14^{\circ}$ dia, em consonância com as fases da cicatrização, indicando um maior nível na fase proliferativa, com alta síntese de colágeno e acelerado processo cicatrizante da ferida (LATIF et al., 2015).

\subsubsection{Atividade antimicrobiana}

A atividade antimicrobiana é relevante ao processo de cicatrização pois as feridas cutâneas estão expostas ao ambiente e neste é possível que ocorra a contaminação por microrganismos como Escherichia coli, Staphylococcus aureus e Streptococcus faecalis. A contaminação de uma ferida pode retardar a cicatrização, além de acarretar em outras complicações, nesse sentido, a Amendoeira-dapraia e a flor da Opúncia apresentam uma atividade antibacteriana e antifúngica que pode prevenir infecções nos ferimentos, possibilitando uma melhor cicatriz e mais rápida (KHAN et al., 2014; AMMAR et al., 2015).

\subsubsection{Produção de colágeno}

A produção do colágeno também é muito importante para a cicatrização, nesse ponto, a Aloe vera mostrou-se uma planta com uma capacidade de estimular a produção de colágeno por intermédio da interação com o genoma das células, os genes MMP-3 e TIMP-2 tiveram a sua expressão regulada pela planta durante o processo de cicatrização, o que provavelmente teve influência em várias etapas da cicatrização, tanto na formação do tecido de granulação como no próprio fechamento da ferida (TABANDEH; ORYAN; MOHAMMADALIPOUR, 2014).

\subsubsection{Proliferação de vasos sanguíneos}

$\mathrm{O}$ aumento do número de vasos sanguíneos que ocorrem no tecido em cicatrização contribui para que a ferida tenha boa oxigenação, além disso, influencia também no aumento da produção de colágeno e permiti uma maior proliferação celular, a análise desse marcador é feito pela contagem de vasos que aparecem por milímetro quadrado. Dessa forma, plantas como a Embaúba (Cecropia pachystachya) e a Pistacia atlantica apresentam um estimulo para a produção de vasos sanguíneos, o que permite com que a cicatrização do tecido epitelial ocorra de forma mais rápida (FARAHPOUR et al., 2015; DUQUE et al., 2016).

\section{CONSIDERAÇÕES FINAIS}

Foi possível avaliar os diversos aspectos em que as plantas medicinais acabam influenciando o processo de cicatrização, como na redução do tempo de cicatrização por meio da estimulação na produção de colágeno e enzimas 
antioxidantes, além disso, a atividade bactericida também é um ponto importante dentre os efeitos das plantas.

Além disso, há relevância do fato de que existe várias plantas de diferentes partes do mundo, o que reflete a influência do

\section{REFERÊNCIAS}

AMMAR, I. et al. Antioxidant, antibacterial and in vivo dermal wound healing effects of Opuntia flower extracts. Int J Biol Macromol. v. 81, p. 483-490. 2015.

BEGASHAW, B. et al. Methanol leaves extract Hibiscus micranthus Linn exhibited antibacterial and wound healing activities. BMC Complement Altern Med. v. 17, n. 1, p. 337.2017.

BERGMAN, I.; LOXLEY, R. Two improved and simplified methods for the spectrophotometric determination of hydroxyproline. Anal. Chem. v. 35, n. 12, p. 1961-1965. 1961.

BHANDIRGE, S.K. et al. Evaluation of Wound Healing Activity of Ethanolic Extract of Pongamia pinnata Bark. Drug Res (Stuttg). v. 65, n. 6, p. 296299. 2015.

BHAT, P.B. et al.Evaluation of wound healing property of Caesalpinia mimosoides Lam. J Ethnopharmacol. v. 193, p. 712-724. 2016.

CHAN, B. et al. Cost-of-illness studies in chronic ulcers: a systematic review. J Wound Care. v. 26, suppl. 4, p. S4-S14. 2017.

CHANDRA, P. et al. Protective effect of Lygodium flexuosum (family: Lygodiaceae) against excision, incision and dead space wounds models in experimental rats. Toxicol Ind Health. v. 31, n. 3, p. 274-280. 2015.

DEMIDOVA-RICE; T.N.; HAMBLIN, M.R.; HERMAN, I.M. Acute and impaired wound healing: pathophysiology and current methods for drug delivery, part 1: normal and chronic wounds: biology, causes, and approaches to care. Adv Skin Wound Care. v. 25, n. 7, p. 304-314. 2012.

DUQUE, A.P. et al. In vivo wound healing activity of gels containing Cecropia pachystachya leaves. J Pharm Pharmacol. v. 68, p. 128-138. 2016.

EMING A.S.; MARTIN P.; TOMIC-CANIC, M. Wound repair and regeneration: mechanisms, signaling, and translation. Sci Transl Med. v. 6, n. 265, p. $265 \mathrm{sr} 6.2014$. conhecimento popular sobre a medicina, e estas plantas apresentam um bom potencial como cicatrizante, porém, ainda é necessário o desenvolvimento de maior número de pesquisas a fim de trazer a utilização dessas plantas para a prática clínica.

ERGENE, Ö. B. et al. Wound healing and antiinflammatory activity of some Ononis taxons. Biomed Pharmacother. v. 91, p. 1096-1105. 2017.

FARAHPOUR, M. R. et al. Hydroethanolic Pistacia atlantica hulls extract improved wound healing process; evidence for mast cells infiltration, angiogenesis and RNA stability. Int J Surg. v. 17, p. 88-98. 2015.

GUO, S.; DIPIETRO, L.A. Factors affecting wound healing. J Dent Res. v. 89, n. 3, p. 219-229. 2010.

IQBAL, A. et al. Management of Chronic Nonhealing Wounds by Hirudotherapy. World J Plast Surg. v. 6, n. 1, p. 9-17. 2017.

JÄRBRINK, K. et al. The humanistic and economic burden of chronic wounds: a protocol for a systematic review. Syst Rev. v. 6, n. 1, p. 15. 2017.

KHAN, A. A et al.Evaluation of Wound Healing Property of Terminalia catappa on Excision Wound Models in Wistar Rats. Drug Res (Stuttg). v. 64, n. 5, p. 225-228. 2014.

KOVÁČ, I. et al. Plantago lanceolata L. water extract induces transition of fibroblasts into myofibroblasts and increases tensile strength of healing skin wounds. J Pharm Pharmacol. v. 67, n. 1, p. 117-25. 2015.

KTARI, N. et al. Antioxidant and hemolytic activities, and effects in rat cutaneous wound healing of a novel polysaccharide from fenugreek (Trigonella foenumgraecum) seeds. Int J Biol Macromol. v. 95, p. 625634. 2017.

LATIF, M.A. et al. Alocasia denudata Engler treatment enhance open wound healing activities in Wistar rat's skin. J Ethnopharmacol. v. 176, p. 25867. 2015.

MOGHADAMTOUSI, S.Z. et al. Annona muricata leaves accelerate wound healing in rats via involvement of Hsp70 and antioxidant defence. Int J Surg. v. 18, p. 110-117. 2015.

NUSSBAUM, S. R. et al. An Economic Evaluation of the Impact, Cost, and Medicare Policy Implications of Chronic Nonhealing Wounds. Value Health. v. 21, n. 1, p. 27-32. 2018. 
ÖZBILGIN, S. et al. In vivo wound-healing activity of Euphorbia characias subsp. wulfenii: Isolation and quantification of quercetin glycosides as bioactive compounds. J Ethnopharmacol. v. 224, p. 400-408. 2018.

PANDA, V.; THAKUR, T. Wound healing activity of the inflorescence of Typha elephantina (Cattail). Int $\mathbf{J}$ Low Extrem Wounds. v. 13, n. 1, p. 50-57. 2014.

SUGUMAR, S. et al. Ultrasonic emulsification of eucalyptus oil nanoemulsion: antibacterial activity against Staphylococcus aureus and wound healing activity in Wistar rats. Ultrason Sonochem. v. 21, n. 3, p. 1044-1049. 2014

TABANDEH, M.R.; ORYAN, A.; MOHAMMADALIPOUR, A. Polysaccharides of Aloe vera induce MMP-3 and TIMP-2 gene expression during the skin wound repair of rat. Int $\mathbf{J}$ Biol Macromol. v. 65, p. 424-430. 2014.

TSALA, D.E. et al. Evaluation of the antioxidant activity and the healing action of the ethanol extract of Calotropis procera bark against surgical wounds. J Intercult Ethnopharmacol. v. 4, n. 1, p. 64-69. 2015.

TÜMEN, İ. et al. Research on the antioxidant, wound healing, and anti-inflammatory activities and the phytochemical composition of maritime pine (Pinus pinaster Ait). J Ethnopharmacol. v. 211, p. 235-246. 2018.

UPADHYAY, A. et al. Topical application of Cleome viscosa increases the expression of basic fibroblast growth factor and type III collagen in rat cutaneous wound. Biomed Res Int. v. 2014, p. 680879. 2014.

YADAV, E. et al. Attenuation of dermal wounds via downregulating oxidative stress and inflammatory markers by protocatechuic acid rich n-butanol fraction of Trianthema portulacastrum Linn. in wistar albino rats. Biomed Pharmacother. v. 96, p. 86-97. 2017.

\section{Tereza Cristina dos Reis Ferreira}

Fisioterapeuta e professora da Universidade do Estado do Pará

João Pedro dos Reis da Costa
Graduando do bacharelado de medicina da
Universidade do Estado do Pará

Beatriz Sayuri Vieira Ishigaki

Graduando do bacharelado de medicina da Universidade do Estado do Pará
Eduardo Augusto Silva Monteiro

Graduando do bacharelado de medicina da Universidade do Estado do Pará 